Review Article

doi:10.29052/IJEHSR.v6.i1.2018.39-46

\title{
Reducing physical restraint use in critically ill patients
}

Sona Duwadi, Birendra Singh Budal ${ }^{2}$ \& Zhao Qing Hua ${ }^{1}$

${ }^{1}$ School of Nursing, The First Affiliated Hospital of Chongqing Medical University

${ }^{2}$ Department of Anesthesiology, Seti Zonal Hospital, Nepal

Corresponding Author Email: qh20063@163.com, sonaduwadi@yahoo.com

Received 25/2/2018; Accepted 30/3/2018; Published 31/3/2018

\section{Abstract}

Background: Patients in critical care setting often encounter severe delirium and agitation putting them at risk of harm. So it has become a common trend to restraint patient physically in intensive care units. However, physical restraint has several adverse physical, psychological and ethical consequences. Awareness regarding significant risk associated with physical restraint, continuing education and skills stabilize the patient's safety and potential complication regarding ethical and legal issues to physical restraint in critical care settings. This study aims to address if any alternatives have been tested regarding physical restraint to improve the patient care for critical care.

Methodology: A search conducted using the PubMed, Medline and Google Scholar including physical restraint use in critical care setting from 1994 to 2017. Studies published in English were included whereas articles published in psychiatric care, children and outside the hospital were excluded. The keywords used were critical care, critically ill, ICU patient safety and physical restraint. The Boolean phrases were used to clarify the quality of search results: "physical restraint \& critical care," "physical restraint \& critically ill" “'physical restraint \& ICU,', physical restraint \& patient safety.' Additionally, reference lists of selected papers were then evaluated further.

Results: Enhanced knowledge, focused education and continuing training to improve skills in using restraint alternatives, awareness of the risk, early identification, conduct proper institutional policies and guidelines, and proactive intervention is the significant measures in alleviating physical restraint use in critical care settings.

Conclusion: A restraint-free environment not only promotes patient safety but also ensure greater caregivers' job satisfaction reducing ethical issues.

\section{Keywords}

Critical care, critically ill, ICU, Patient safety, Physical restraint

\section{Introduction}

Physical restraint (PR) is a manual or mechanical device, material, or equipment attached to a patient's body that restricts movement or regular access. PR is highly used in the critical care setting, up to the range of $75 \%$ in a mechanically ventilated patient at least once during their intensive care unit admission ${ }^{1}$. World widely, PR use range from $3.4 \%$ to $21 \%$ for $2.7-4.5$ days $^{2}$. In the US, approximately 27,000 patients are restrained physically every day in health care setting ${ }^{3}$. Moreover, PR was used most frequently in South African Hospital's critical care setting with prevalence $50 \%{ }^{4}$ and corresponded with Huang's findings between $39.1 \%$ and $69.9 \%$ in Taiwan ${ }^{5}$. Recently, Canadian ICU reported the prevalence rate of PR use ranges from $53 \%$ to $79 \%{ }^{6}$. Clinical justification to use PR in ICUs is to support ongoing treatment such as accessing invasive medical devices to avoid unplanned treatment intrusion and potentially life-threatening consequences promoting safety and desired prognosis ${ }^{4,7,8}$. In contrast, PR use in the ICUs is questionable globally ${ }^{9}$ because research has shown that restrained patient encounter prolongs hospital stays and complications results from immobility ${ }^{7}$, adverse psychological consequences like agitation, 
delirium and possibly lethal complication of critical illness ${ }^{1,10}$. Indeed, because of pre-existing medical conditions ${ }^{11}$, critically ill patients itself are highly at risk for the development of agitation and delirium ${ }^{2}$ which has been suggested that the prevalence of these problems in ICUs varies hugely in the literature from $15 \%$ to $87 \%{ }^{11}$.

Furthermore, PR can be sensed as an ethical dilemma, conflicting norms, values and interfering patient's autonomy ${ }^{12}$. Various policy and guideline reports propose to lessen physical restraint practice variability and its use 8,13,14 such as Ontario' $\mathrm{s}$ Patient Restraints Minimization Act in Canada, which constitutes restraint reduction for patient safety $^{14}$. Rainier studied alternatives to using restraints for alcohol-withdrawal patients, and he noted that education and culture change had the only substantial effect in reducing restraint use ${ }^{7}$. Using best practices to educate health care providers about restraint overuse is one efficient way to influence caregivers' decision-making positively ${ }^{14}$. For instance, one-to-one education grants opportunities to explore nurses' perceptions of alternatives to physical restraints ${ }^{15}$. Aware of the significant risks associated with the use of physical restraint is supporting for changes in practice. In spite of these identified trends, reduction of PR use is proving to be a perplexing matter. Unfortunately, less evidence-based guidance on practices to minimize restraint use are available due to the paucity and unmet quality of existing studies. The identification of effective PR reduction policies is a clinical and research crucial and implies caregivers, patient and family reported experience and outcome measures. To state this requirement, this article aimed to study the available literature on alternatives to PR in critically ill patients to provide evidenced-based nursing practice in reducing restraint use in this particular patient population.

\section{Influences ICU caregiver's use of physical restraint}

When addressing alternatives to PR, it is necessary to understand why clinicians choose to place PR. There is no doubt about the use of restraint therapies in the ICUs is nonetheless for patient safety which minimizes the risk of intentional as well as accidental extraction of medical devices. Also, aid to perform bedside procedures for the noncooperative patient. Following a comprehensive study of the literature within the critical care settings regarding the PR, its use, alternatives, and treatment interference $^{9}, 11,12,15-20$, the choice to restraint usually based on life-threatening consequences occurring from the opposition with treatment therapies. E.g., pulmonary artery catheters, endotracheal tubes, urinary drainage catheter, and interference with monitoring leads, intravenous lines and blood pressure cuffs.

It is estimated that up to $74 \%$ of patient in ICUs are restrained physically at device disruption time ${ }^{3}$. Conversely, Nurses' characteristics; her working experience, knowledge of expected outcomes resulting from implementation of a plan of action, a decision for one ethical belief over another (e.g., beneficence vs. autonomy) and environmental aspects like nurse-patient ratio and workload are other determinants that initiate nurses to use $\mathrm{PR}^{21}$. To support this statement, an observational study by Martin \& Mathisen on 50 ICU patients in the USA and 50 ICU patients in Norway determined that because of inadequate nurse-patient ratio, there could be the reason for using PR profoundly ${ }^{22}$. To use PR whatever choice the nurse makes, or to practice some different plan of action, it results in a subsequent patient, nurse-related and institutionrelated outcomes ${ }^{18}$. A descriptive study by Minnick et al. ${ }^{9}$ examined the prevalence of PR throughout the United States from 40 randomly selected acute care hospitals in 6 different urban areas and affirmed that the most usually documented purpose for PR was to "prevent therapy disruption" (74.9\%), "confusion" (25.4\%) and "fall prevention" (17.6\%). Furthermore, a prospective observational study by Luk By et al. determined that the several common reasons for critically ill patients to restraint physically are agitation (43\%), restlessness (17\%) and preventive means to accidental device extraction and maintain patient safety $(17 \%)^{6}$. Therefore, the reason to PR for ICU nurses is to maintain the patients' treatment devices and therapies, such as mechanical ventilation, 
intravenous line, urinary catheter etc. to prevent termination of life-sustaining therapies.

\section{Alternatives}

Whilst, some alternatives to PR in the ICU have been introduced; it is not prospectively appraised regarding the effectiveness and safety of those intercessions. Per se, maximum of these studies have been carried outside the critical care setting, and the pertinence of these alternatives to restraints remains unverified for critically ill patients. A further scheme is an identification of cause that can alter patient's discomfort and agitation that reinforce nurses to restraint patient. For example, correction of endotracheal tube malposition, pain related to intravenous line, distress associated with a urinary catheter, etc. help to limit agitation ${ }^{8}$.

An additional, the practices of diversional therapies that also may mitigate the agitation level. These include music, involving family members to access the patient, reorientation to the situation, giving personal attention to the patient, alter ICU environment decreasing agitation producing stimuli, reduce noise, use of bed exit alarm, comfort patient with relieving pain and providing emotional care that entails lessening the use of restraints ${ }^{8,19,21,23-26}$. As we mentioned above, the patient with PR use may suffer from agitation, delirium, loss of dignity, emotional distress, a complication associated with immobility and mostly posttraumatic stress disorder results with restraint use which not only interfere physically but also psychological even after recovery from critical illness. Taking those matters into consideration, in an acute and critical care setting, Joint Commission on Accreditation of Health Care Organization (JCAHO) standard of care limits the use of physical restraint, which carried various measures. Frequent reorientation, continuous observation, pharmacological measures, recreation, satisfying control of pain, stabilize and secure medical devices, reassuring the patient results in managing delirium and agitation thus restrict $P R$ to use $\mathrm{e}^{18,27}$.
Consequently, for reasonable patient safety, it is needed to focus on multidisciplinary education on the use of PR, its prevention and ongoing evaluation of outcomes ${ }^{4}$. An intervention by Cosper et al. developed an execution of multidisciplinary inspection on the patient with restraint and expanded accessibility of restraint alternatives as providing education regarding restraint to health care providers, and this program has maintained a periodical restraint prevalence rate less than $2.26 \%{ }^{24}$. Since before, in critical care settings, restraint has been used to prevent patients from being harmed themselves by interfering therapeutic devices.

So, to change this practice through education regarding indication and alternatives for restraint, patients' right, proper recording, and reporting that might assist in decision making on the use of $\mathrm{PR}^{15}$. In 2009, Huang et al. studied the efficacy of focused education on nurses' attitudes and practiced on the use of restraint. After the evaluation of pretest/posttest, the investigators noticed with ameliorated knowledge and skill on nursing staffs and perceived positive attitude towards the use of restraint alternatives that later on led to lowered restraint prevalence 5 . On the same line, a quasiexperimental study design was carried out by Taha \& Ali and Yen et al., in Zagazig University Hospital, Egypt, and Southern Taiwan Medical Centre in ICU respectively ${ }^{28,} 29$. Both of these studies were conducted to appraise the effectiveness of education and training intervention regarding nurses' knowledge and their practice of restraint use. Pre and post-test were taken, and the result showed the significant improvement in nurses' knowledge and attitude towards using restraint which aids in enhancing the quality of care contributed to critically ill patients ${ }^{28,29}$.

These verdicts indicated the decisive impact regarding advancing education and practice on nurses' knowledge of PR while lacking knowledge about the use of restraints limits a nurse's ability to consider the need and implement proper care. In fact, constant education provides nurses with a 
genuine perception of restraints and dispels common misconceptions regarding their use. Furthermore, in one study; conducted an evidencebased practice standard to minimize restraint with the purpose of creating restraint-free environment via education, training intervention to staffs and patient-centered periodic treatment activities to promote personal growth and self- esteem. This follows in meaningful results including $30 \%$ reduction in restrained and $55 \%$ in restraints hours $^{30}$. Besides, Hall et al., ${ }^{10}$ developed an evidence-based project called Restraint Management Bundle (RMB) program in critical care setting which was intended to minimize restraint, educating staffs and upgrade patient's quality and safety. Along with, interdisciplinary team members designed a patient-centric strategy to reduce restraint use by patient's characteristics. Outcomes showed that the proportion of ICU patients' restrained dropped significantly ( $24.3 \%$ vs. $20.9 \%$ ) accompanying the implementation of a program.

This RMB project succinct provides a frame to manage in reducing restraint, which minimizes harm and gains patient safety ${ }^{10}$. Another Canadian study presented that there is increased ICU nurses' knowledge after the advancing education of the evidence regarding restraint ${ }^{24}$ through a PR training plan appeared statistically notable reduction in the use of restraint after one year ${ }^{14}$. Consequently, researchers concluded that program regarding PR with an educational component, restraint removal, individualized specific need, and interventions are the active components in lowering restraint use which revealed that educational programs had a positive impact on nurses' knowledge, attitude and practice regarding the use of $\mathrm{PR}^{5,22,23,25,31,32}$.

\section{Ethical Consideration}

Critically ill patients are tentatively unable to care for themselves and make their own decisions regarding their requirements, wishes, and values ${ }^{7,8}$, $13,22,33-37$ because of their disease condition. Those patient pose a hurdle to nurses and healthcare providers in providing care with verbal and non- verbal communication, memory loss, safety, physical functioning, nutrition, motor difficulty, social isolation. Misinterpretation of this kind of role advocacy can drive to nurses' inappropriate behavior and cause ethical problems in nursing practice $^{17}$. PR is in direct conflict with the notion of autonomy as it poses ethical dilemmas for nursing staff. It has a dehumanizing influence on both nurses and the patient, which has an intense impact on the total caring process $5,7,16,17$.

Although the patient is controlled regardless of his/her will, values needs; he/she has right to autonomy and quality health care whereas the team members of care providers have right to work on the safe environment as well. When applying PR, nurses are manifested with the ethical dilemmas related to autonomy and patient safety. In spite of this controversy, PR continues to be involved in critical care settings ${ }^{5}$. Restraint patient experience feeling of guilt, embarrassment and a loss of dignity $^{34,36,38}$. The medical and nursing ethics both affirm respect for the autonomy and patient's dignity. It can be improved by evolving a therapeutic relationship between care providers and patient that is usually an essential, trustworthy ethical relationship between nurses and patients. In the same way, inappropriate use of PR needlessly exposes the patient to a risk of injury and other potential complications.

Therefore, it violates the principle of autonomy (right to take a decision), beneficence (doing well) and non-maleficence (avoiding harming) 11,19,34,37 . It is the nurses, and caregiver's responsibility to respect patient's autonomy but the decision to use PR disrupts the principle of informed consent ${ }^{11}$. It is inferred that caregivers, clinicians, and nurses should look for alternatives, knowing the ethical dilemmas that arise while deciding restraint. In such situation, the primary intent for nurses is patient safety. Although suitable PR alternative is used, nurses can maintain the patient safety without breaking patient's autonomy ${ }^{8}$. Nonetheless, when a client seeks treatment, it is reasonable to consider implied consent when a patient and patient's 
delegate is unable to provide consent, care providers might give restraint therapies in a relevant situation. Additionally, patient's injurious deeds that a violate patient might commit unknowingly; an ethical concern is paramount to shield the medical team and patient as well. On the other hand, incoherent but conscious patients are cognizant of restraint and find them discomfort. In this circumstances, the dilemma results of balancing discomfort vs. medical necessity. The medical team usually present with such situation that is both ethically ambiguous as well as psychologically offensive. The standard that most health professionals cohere is that of no maleficence, beneficence and respect patient's autonomy. Even though the reason to place PR is often to preserve the patients from their destructive actions, the studies imply that restraint is more detrimental than beneficial $^{7,16}$.

\section{Nurses' Role}

Generally speaking, nursing is the highly trusted profession by the public as nurses are almost involved in caring patient, responsible for maintaining patient's safety including critically ill and decision making when a frequent absence of medical orders in starting and removing PR. Nurses are the ones who are accountable for adjusting the plan of care based on periodic assessment according to the patient's response and releasing the restraint. Furthermore, nurses should find out the additional causes of agitation and treat accordingly, let the patient's party know about the necessity for restraint and reconsider medical orders frequently in critical care settings ${ }^{8,28}$. In one study of critical care nurses, discussion and interdisciplinary appraisals regarding the need for PR in critically ill patient were sensed as unnecessary ${ }^{21}$.

Nurses often make the decision when there is the inadequacy of interdisciplinary analysis with patients and families. In contrast, such decision lays an undue burden on the nurses as the individual decision maker and despoils the principles of informed consent. Sometimes, it may arise circumstances where the indication for using PR is relatively clear. Being a Nurse, one should weigh the risks, benefits, and alternatives to PR to consider ethical values. Analyzing the precipitating problems is the first step to be taken. For instance, environmental factors like improper light, noise in the room, patient's factors such as pain, poor vision, and hearing, dysuria, and constipation are triggering the behavior of the patients? does the patient have another associated medical illness? is an adverse reaction to drugs a contributing factor? PR nevermore fixes the underlying problem; addressing the cause behind the patient's triggered behavior is the critical factor in soothing the patient. In everyday nursing practice, being presentative, communicative, providing meaningful activities, responsible, changing the environment according to the need of the patient is the nursing, primary intervention. Those interventions not only give patients a feeling of safety and comfort but also potentially to nurses feeling better insight towards what is happening to the patients. Thus, develops a good rapport between patient and nurse and enable patient to think that nurses are supporting and understanding the patients' experiences. To tackle such condition, nurses need to be educated to deal with aggressive behavior patient and implement safe environment using effective alternative to PR. A wider understanding towards clinical decisionmaking is the crucial factor in helping nurses to implement policies and safe practices regarding patients at risk and enhances skills and knowledge to assist an interdisciplinary team to prevent the use of $\mathrm{PR}^{39}$.

A thorough inter-professional discussion should begin to reduce the risk of restraint use. Continuing assessment and monitoring changes in patient's physical and cognitive functional abilities can alter nurses' feeling to use restraint for safety. Including such issues on educational policies for nurses such as continued in-service training on PR use. Educational and understanding level of nurses in using PR, its alternatives as well as institutional policy influence the frequency of PR applied ${ }^{17}$. Addressing the concerns regarding institutional policies, a complication that arises from restraint use, inadequate restraint knowledge and 
misconception on their use among nurses helps in finding the cause of the potential problem which also helps to promote patient care ${ }^{29}$.

An American study showed that the nurses' having inadequate knowledge regarding restraint and later on with education changed their perception towards using restraint which leads to $60 \%$ decline in its use $^{11}$. Likewise, a study proposed that enhanced knowledge, attitude, and skills of nurses via inservice training on PR, advancing guidelines/policies and providing sufficient staffs as well as equipment helps to sustain patient's safety and prevent complication ${ }^{2}$. Therefore, nurses with adequate education and skills can play a significant role in stabilizing the patient's safety and consequences regarding the ethical and legal problems related to PR in critical care settings.

\section{Future Prospects}

Enhanced efforts are necessary to better understand the standard guidelines and policies in practicing and minimizing PR. Strategies such as orientation programs for newly joined nurses, free booklets, and manual regarding PR in each restraint using critical care settings in the hospital to promote the care providers' skills and knowledge along with regular supervision and feedback. Moreover, the available literature on the use of PR has emphasized a various number of vital issues to consider in using restraint within intensive care settings. Supplementary research in this area may contribute further guidance on the consequences of coercive intervention for both 'receivers' and 'caregivers.'

Focused educational program on the causes of agitation and delirium in a patient, which initiates them for violent behavior. Instead, to take action by restraining to control such activities in a patient, it is better to prevent the causative factors, which provoke the activities. Managing a critically ill patient with effective communication, reorientation, comfort them by maintaining proper environment by minimizing the light and noise, allow interacting with family members helps them to calm the situation. In one hand, educational programs on the facts and myths about using restraint, alternative measures to restraints, decision-making process, adverse impacts on using PR, managing violent behavior and minimizing restraint use increase positive patient outcomes whereas in other hands there is greater nurse job satisfaction too. The ongoing expansion of alternative clinical interventions that direct on particular competency and definite skill facilitation has also become a significant research preference.

This includes expansion of the literature on collaboration and facilitation on behavioral intervention, as it is essential in educating all caregivers on a behavioral concept. Here, behavior means the especially highly unstable situation of the patient which is considered as the reason for restraining. Knowing and addressing the causative factor is vital to care providers and patient safety as well. To best implementation, continuing education is necessary for all healthcare providers in reaching the proper conclusion and desired change regarding restraint use minimization. Hence, with education, awareness of the risk, early identification, institutional policies and pro-active intervention are the essential elements for caregivers in distinguishing proper alternatives and reducing the coercive procedures like PR.

\section{Strength and Limitation}

This study comprised of a number of articles concerning PR, a decision in using restraint, its alternatives, ethical issues and nurses' intervention in using PR in critically ill patients that may contribute further guidance on the consequences of coercive intervention for both patients and caregivers in maintaining the restraint-free environment. The study has limitations as well. The study included critically ill patient, but it has not a well-distinguished age group. Besides, a lack of research regarding the effectiveness of particular alternatives on reducing PR, it is unidentified the nursing specific alternative intervention to restraint and their efficacy in a critically ill patient. 


\section{Conclusion}

Despite using PR in critical care settings is common, there is little research that examines its reduction. As we went through available literature, few studies that discuss the issue and suggest using alternatives to restraint but does not reflect statistical evidence regarding their effectiveness in minimizing restraint rates. Besides, the alternatives mentioned above are considered more relevant for critically ill patients in a state of agitation and delirium. Therefore, educating staffs focused on improving skills, awareness of the risk, early identification, guidelines and proactive intervention is paramount to ensure restraint-free environment. Even though our study has been known as an area of concern, further prospective and randomized controlled trial on identifying the nursing specific alternative intervention to restraint and their effectiveness on the reducing $\mathrm{PR}$ among critically ill patient to maintain patient and staff safety is desirable.

\section{Conflict of Interest}

None.

\section{Acknowledgment}

It is a concern of pleasure for me to express heartfelt gratitude towards all those who directly/indirectly supported me to accomplish my goals. Most importantly, I would like to express thanks to my parents, sisters and a beloved husband who are an immense source of strength and inspiration.

\section{Funding}

None.

\section{References}

1. Rose L, Dale C, Smith OM, Burry L, Enright G, Fergusson D, et al. A mixed-methods systematic review protocol to examine the use of physical restraint with critically ill adults and strategies for minimizing their use. Syst Rev. 2016;5(1):194.

2. Suliman M, Aloush S, Al-Awamreh K. Knowledge, attitude and practice of intensive care unit nurses about physical restraint. Nurs Crit Care. 2017;22(5):264-9.

3. Mion LC. Physical restraint in critical care settings: will they go away? Geriatr Nurs. 2008;29(6):421-3.
4. Kirk AP, McGlinsey A, Beckett A, Rudd P, Arbour R. Restraint Reduction, Restraint Elimination, and Best Practice: Role of the Clinical Nurse Specialist in Patient Safety. Clin Nurse Spec. 2015;29(6):3218.

5. Huang HT, Chuang YH, Chiang KF. Nurses' physical restraint knowledge, attitudes, and practices: the effectiveness of an in-service education program. J Nurs Res. 2009;17(4):241-8.

6. Luk E, Burry L, Rezaie S, Mehta S, Rose L. Critical care nurses' decisions regarding physical restraints in two Canadian ICUs: A prospective observational study. Can J Crit Care Nurs. 2015;26(4):16-22.

7. Rainier NC. Reducing physical restraint use in alcohol withdrawal patients: a literature review. Dimensions of critical care nursing : DCCN. 2014;33(4):201-6.

8. Maccioli GA, Dorman T, Brown BR, Mazuski JE, McLean BA, Kuszaj JM, et al. Clinical practice guidelines for the maintenance of patient physical safety in the intensive care unit: use of restraining therapies--American College of Critical Care Medicine Task Force 2001-2002. Crit Care Med. 2003;31(11):2665-76.

9. Minnick AF, Mion LC, Johnson ME, Catrambone C, Leipzig R. Prevalence and variation of physical restraint use in acute care settings in the US. J Nurs Scholarsh. 2007;39(1):30-7.

10. Hall DK, Zimbro KS, Maduro RS, Petrovitch D, Ver Schneider P, Morgan M. Impact of a Restraint Management Bundle on Restraint Use in an Intensive Care Unit. J Nurs Care Qual. 2017.

11. Hine $\mathrm{K}$. The use of physical restraint in critical care. Nurs Crit Care. 2007;12(1):6-11.

12. Luk E, Sneyers B, Rose L, Perreault MM, Williamson DR, Mehta S, et al. Predictors of physical restraint use in Canadian intensive care units. Crit Care. 2014;18(2):R46.

13. Bray K, Hill K, Robson W, Leaver G, Walker N, O'Leary M, et al. British Association of Critical Care Nurses position statement on the use of restraint in adult critical care units. Nurs Crit Care. 2004;9(5):199-212.

14. Hurlock-Chorostecki C, Kielb C. Knot-So-Fast: a learning plan to minimize patient restraint in critical care. Dynamics. 2006;17(3):12-8.

15. Hevener S, Rickabaugh B, Marsh T. Using a Decision Wheel to Reduce Use of Restraints in a 
Medical-Surgical Intensive Care Unit. Am J Crit Care. 2016;25(6):479-86.

16. Goethals S, Dierckx de Casterle B, Gastmans C. Nurses' decision-making in cases of physical restraint: a synthesis of qualitative evidence. J Adv Nurs. 2012;68(6):1198-210.

17. Liukkonen A, Laitinen P. Reasons for uses of physical restraint and alternatives to them in geriatric nursing: a questionnaire study among nursing staff. J Adv Nurs. 1994;19(6):1082-7.

18. Mion LC. Establishing alternatives to physical restraints in the acute care setting: a conceptual framework to assist nurses' decision making. AACN Clin Issues. 1996;7(4):592-602.

19. Kapp MB. Physical restraint use in critical care: legal issues. AACN Clin Issues. 1996;7(4):579-84.

20. Vance DL. Effect of a treatment interference protocol on clinical decision making for restraint use in the intensive care unit: a pilot study. AACN Clin Issues. 2003;14(1):82-91.

21. Reigle J. The ethics of physical restraints in critical care. AACN Clin Issues. 1996;7(4):585-91.

22. Martin B, Mathisen L. Use of physical restraints in adult critical care: a bicultural study. Am J Crit Care. 2005;14(2):133-42.

23. Molasiotis A. Use of physical restraints. 2: Alternatives. Br J Nurs. 1995;4(4):201-2, 19-20.

24. Cosper P, Morelock V, Provine B. Please release me: restraint reduction initiative in a health care system. J Nurs Care Qual. 2015;30(1):16-23.

25. Said AA, Kautz DD. Reducing restraint use for older adults in acute care. Nursing. 2013;43(12):59-61.

26. Markwell SK. Long-term restraint reduction: one hospital's experience with restraint alternatives. J Nurs Care Qual. 2005;20(3):253-60.

27. Zhang Z, Pan L, Deng H, Ni H, Xu X. Prediction of delirium in critically ill patients with elevated Creactive protein. J Crit Care. 2014;29(1):88-92.

28. Zeinab H Ali NMT. Physical Restraints in Critical Care Units: Impact of a Training Program on Nurses' Knowledge and Practice and on Patients' Outcomes. Journal of Nursing \& Care. 2013;02(02).

29. Yeh SH, Hsiao CY, Ho TH, Chiang MC, Lin LW, Hsu CY, et al. The effects of continuing education in restraint reduction on novice nurses in intensive care units. J Nurs Res. 2004;12(3):246-56.
30. Huckshorn KA, LeBel J, Jacobs HE. An organizational approach to reducing and preventing restraint and seclusion use with people with acquired brain injury. NeuroRehabilitation. 2014;34(4):67180.

31. Godkin MD, Onyskiw JE. A systematic overview of interventions to reduce physical restraint use in longterm care settings. Online J Knowl Synth Nurs. 1999;6:6.

32. Johnson K, Curry V, Steubing A, Diana S, McCray A, McFarren A, et al. A non-pharmacologic approach to decrease restraint use. Intensive Crit Care Nurs. 2016;34:12-9.

33. Schorr CA. Patient-initiated device removal in intensive care units: A national prevalence study. Yearbook of Critical Care Medicine. 2009;2009:270-2.

34. Soininen P, Putkonen H, Joffe G, Korkeila J, Valimaki M. Methodological and ethical challenges in studying patients' perceptions of coercion: a systematic mixed studies review. BMC Psychiatry. 2014; $14: 162$.

35. Kontio R, Valimaki M, Putkonen H, Kuosmanen L, Scott A, Joffe G. Patient restrictions: are there ethical alternatives to seclusion and restraint? Nurs Ethics. 2010;17(1):65-76.

36. Benbenbishty J, Adam S, Endacott R. Physical restraint use in intensive care units across Europe: The PRICE study. Intensive and Critical Care Nursing. 2010;26(5):241-5.

37. Preckel B, Schlack W. Editorial III: xenon-cardiovascularly inert? $\mathrm{Br} \mathrm{J}$ Anaesth. 2004;92(6):786-9.

38. Martin B. Restraint use in acute and critical care settings: changing practice. AACN Clin Issues. 2002;13(2):294-306.

39. Chao CM, Lai CC, Chan KS, Cheng KC, Ho CH, Chen CM, et al. Multidisciplinary interventions and continuous quality improvement to reduce unplanned extubation in adult intensive care units: A 15-year experience. Medicine (Baltimore). 2017;96(27):e6877. 